\title{
12. DNA Recognition Rules for Steroid Hormone Receptors and GATA1 (1): Chemical and Stereochemical Rules
}

\author{
By Masashi SuzuKI*) and Lucy ChothiA \\ MRC Laboratory of Molecular Biology, Hills Road, \\ Cambridge, CB2 2QH, U.K. \\ (Communicated by Setsuro EBASHI, M. J. A., May 12, 1994)
}

\begin{abstract}
DNA recognition rules for transcription factors of class $\mathrm{C} 4$ are discussed in the light of three dimensional structures determined by crystallography or NMR. The rules have two parts; the chemical rules which list possible amino acid side-chains and DNA bases partners, and the stereochemical rules which describe how the positions of the residues and bases which make contact are determined by the orientation and packing of the $\alpha$-helix in the major groove of DNA.
\end{abstract}

Key words: DNA recognition; transcription factor.

1. Introduction. Although the atomic structures of many protein-DNA complexes have shown that recognition usually takes place through $\alpha$-helices (recognition helices) binding in the major groove of $\mathrm{DNA},{ }^{1)}$ the general rules that describe how helices recognise DNA have remained elusive. ${ }^{2)-6)}$ The $\mathrm{Zn}$-binding transcription factors are divided into several classes $\mathrm{C} 2 \mathrm{H} 2, \mathrm{C} 4, \mathrm{C} 6$ according to the type and number of amino acid residues which bind to the $\mathrm{Zn}$; (C-Cys and $\mathrm{H}$-His). In this paper we discuss the $\mathrm{C} 4$ class, following the method used for analysing another type of transcription factors. ${ }^{7,8)}$

Based on an analysis of the atomic structures of the DNA complexes formed by the binding domains of three C4 factors: glucocorticoid receptor ${ }^{9)}[\mathrm{GlcR}]$, oestrogen receptor ${ }^{6)}$ [EstR] and GATA1, ${ }^{10)}$ we propose rules for how the helices of $\mathrm{C} 4$ discriminate between DNA sequences. We show that these rules can successfully account for the DNA-binding of other transcription factors of the C4 class: Tailless ${ }^{11)}$ and HNF4. ${ }^{12)}$

2. Features of the crystal and NMR structures. At the recognition sites of the C4 proteins, a recognition helix makes contact with two strands of DNA. To describe the common features of the sites in the three complexes, the sequences are given the same numbering system (Fig. 1a). We call the DNA strand that runs in the $3^{\prime}$ to $5^{\prime}$ direction, as we proceed along the recognition helix, the Watson(W) strand, and that which runs in the $5^{\prime}$ to $3^{\prime}$ direction, the $\operatorname{Crick}(\mathrm{C})$ strand (Fig. 1a). We show in Figs. 1b-d the contacts between residues and bases that are observed in the DNA complexes of GlcR, ${ }^{9)} \mathrm{EstR}^{6)}$ and GATA1. ${ }^{10)}$ These contacts are summarised in Fig. 1a using solid lines.

Inspection of Fig. 1a reveals a common pattern of contacts in the three complexes. The residues which contact DNA bases mainly come from four positions 1, 4, 5, and 9 . Furthermore, the same base positions are contacted by the same residue positions: the residue aa1 makes contacts with the $\mathrm{W} 2$ base or, in one example, with the $\mathrm{C} 1$ base (see Fig. 1a for the numbering); aa4 with $\mathrm{C} 1$; aa5 with W3, and aa9 with W4. Lys at position 8 is used for base recognition only when its specific partner G is placed at C2 (EstR) or probably at C3 (see section 3).

The reason why these striking similarities in the patterns of the contacts were not

*) Correspondence to: M. Suzuki. 


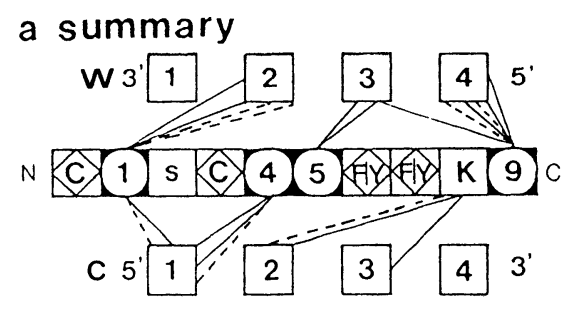

C GICR

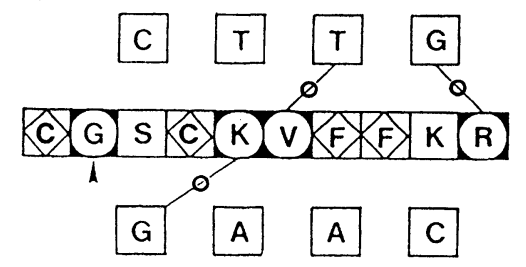

b GATA

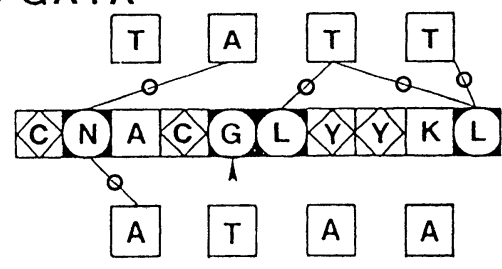

d EstR

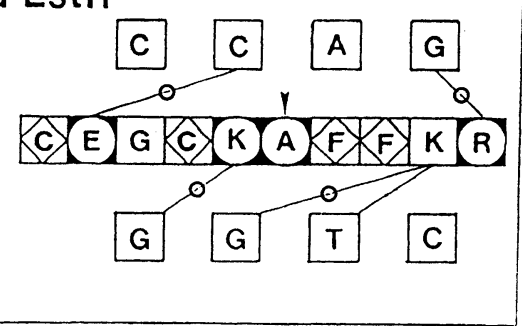

e TLL

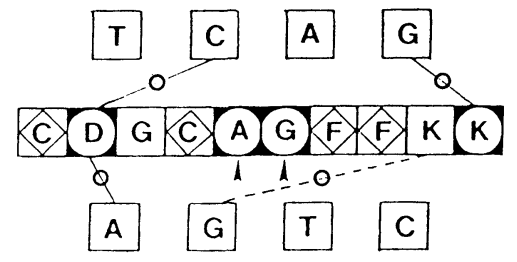

f HNF4

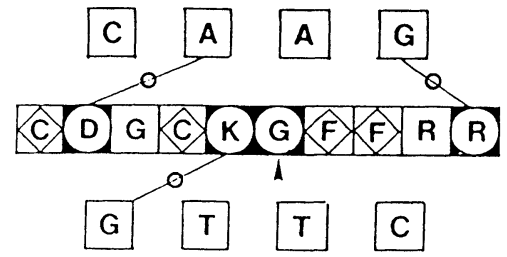

Fig. 1. Observed (b-d) and predicted (e, f), contacts between amino acid and DNA base positions. (a) shows the template. This is a sketch of a recognition helix (centre) binding to the Watson (W) and Crick (C) strands of DNA in the major groove. Solid and broken lines show observed and predicted contacts, respectively. In (b)-(f) circles show "specific" contacts (see Fig. 2). Residues which are placed at the base-recognition positions but are too small to make contact with a DNA base are indicated with arrows.

noticed by earlier workers is, probably, because in each complex one of the 1,4 or 5 positions is occupied by Gly or Ala, which are too small to make contacts to a DNA base, and the position that contains the Gly/Ala is different in the three complexes.

3. Examination of other transcription factors of the $\mathrm{C} 4$ class. The common pattern of DNA-protein contacts in the $\mathrm{C} 4$ structures provides the basis for understanding the rules for DNA recognition by this class of transcription factor. Before discussing these we need to make some general observations on the residues used for the recognition of DNA bases. From a consideration of the potential for good hydrogen bonding or hydrophobic contacts, one of us has put forward a list of which amino acid residues might interact with which DNA bases in the major groove of DNA. ${ }^{8)}$ A concise version of the list (the chemical code) is shown in Fig. 2.

The use of a particular residue at a position in a complex will depend not only upon its intrinsic ability to bind a base but also upon its stereochemical environment, i.e. its ability 


\begin{tabular}{|c|c|c|}
\hline aa & Base & Specificity \\
\hline Ala (s) & $\mathbf{T}$ & ++ \\
\hline $\begin{array}{l}\text { Val (m) } \\
\text { Leu (l) } \\
\text { Ile (m) } \\
\text { Phe (a) }\end{array}$ & $\mathbf{T} / \mathbf{C}$ & + \\
\hline $\begin{array}{l}\text { Met (l) } \\
\operatorname{Trp}(a)\end{array}$ & $\mathbf{T} / \mathrm{C} \geq \mathrm{A}$ & \pm \\
\hline Tyr (a) & $\mathrm{T} / \mathrm{C}>\mathrm{A} / \mathrm{G}$ & \pm \\
\hline $\begin{array}{l}\text { Asp (m) } \\
\text { Glu (l) }\end{array}$ & $\mathbf{C} \geq \mathbf{A}$ & + \\
\hline $\begin{array}{l}\text { Asn (m) } \\
\text { Gln (l) }\end{array}$ & $\mathbf{A}>\mathrm{T} / \mathrm{G} / \mathrm{C}$ & + \\
\hline $\begin{array}{l}\text { Cys (s) } \\
\text { Ser (s) } \\
\text { Thr (s) }\end{array}$ & $\mathrm{A} / \mathrm{T} / \mathrm{G} / \mathrm{C}$ & \\
\hline $\begin{array}{l}\text { Arg (l) } \\
\text { Lys (l) }\end{array}$ & $\mathbf{G} \gg \mathrm{T} \geq \mathrm{A}$ & ++ \\
\hline $\mathrm{His}(\mathrm{m})$ & $\mathbf{G} \geq \mathrm{T} \geq \mathrm{A} \geq \mathrm{C}$ & \pm \\
\hline
\end{tabular}

Fig. 2. Chemical code. The list of possible partners of amino acid sidechains and DNA bases for contacting in the major groove of DNA. The size of the residues, small (s), medium (m), large (l) and aromatic (a) is also shown. The specificity of contact, very high $(++)$, high $(+)$, medium $( \pm)$, and low (the others), is shown. See Ref. 8 for the details.

to pack properly in the protein-DNA interface. In this regard residues involved in recognition can be classified into four groups ${ }^{8}$ : small-Gly, Ala, Cys, Ser, Thr; mediumAsn, Val, Ile, His, Asp; large-Met, Leu, Lys, Arg, Glu, Gln, and a fourth group that consists of aromatic residues. In the C4-DNA complexes the residues used for base-recognition at positions 4 and 9 are large, while those at 1 and 5 , are either medium or large (Fig. 1).

On the basis of the stereochemical rules (Fig. 1a) and of the chemical code (Fig. 2), we can propose a model for the manner in which $\mathrm{C} 4$ transcription factors for which the $3 \mathrm{D}$ structures are as yet unknown but of which the DNA-binding specificity is known, Tailless $^{11)}$ and $\mathrm{HNF}_{4}{ }^{12)}$ recognise particular sequences of DNA. Contacts between residues and bases can be proposed using the rules (Figs. 1e, f).

Many transcription factors of the C4 class have been identified and these have one of the five recognition helices described above and are only different in the way in which they dimerise (see the discussion on the spacing rules in the following paper). Moreover, from the results of the nematode genome project ${ }^{13)}$ a few hormone receptor-like genes have been identified. These have putative recognition helices of different sequences (although we are not certain that the products of these genes really function as a transcription factor). By using the rules it is possible to predict possible binding sites on DNA from their amino acid sequences (Fig. 3). 
a CEL06/SU44

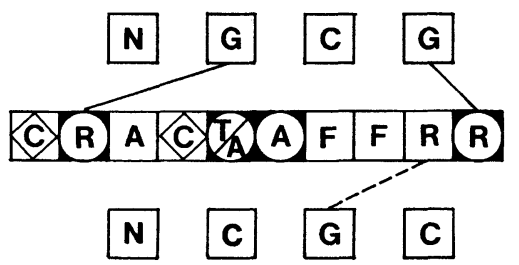

b SV11

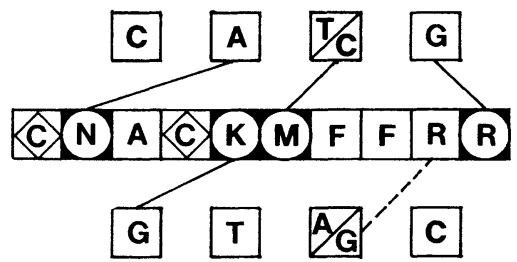

Fig. 3. Prediction of binding sequences of DNA for putative $\mathrm{C} 4$ transcription factors of nematode.

In this paper we have discussed that the chemical and stereochemical rules can explain the contacts observed in the atomic structures and that they can be used for predicting contacts for other $\mathrm{C} 4$ factors.

Acknowledgements. We thank Drs A. Klug and J. Finch, for their critical reading of the manuscript. We thank Dr K. Umesono for informing us of useful literature.

\section{References}

1) Harrison, S.: Nature, 353, 715-719 (1991).

2) Pabo, C. O., and Sauer, R. T.: Annu. Rev. Biochem., 53, 293-321 (1984).

3) Umesono, K., and Evans, R. M.: Cell, 57, 1139-1146 (1989).

4) Mader, S. et al.: Nature, 338, 271-274 (1989).

5) Pabo, C. O., and Sauer, R. T.: Annu. Rev. Biochem., 61, 1053-1095 (1992).

6) Schwabe, J. W. R. et al.: Cell, 75, 567-578 (1993).

7) Suzuki, M.: EMBO J., 12, 3221-3226 (1993).

8) —-: Structure, 2, 317-327 (1994).

9) Luisi, B. F. et al.: Nature, 352, 497-505 (1991).

10) Omichinski, J. G. et al.: Science, 261, 438-446 (1993).

11) Liaw, G.-J. et al.: Proc. Natl. Acad. Sci. U.S.A., 90, 858-862 (1993).

12) Sladek, F. M. et al.: Genes Develop., 4, 2353-2365 (1990).

13) Sulston, J. et al.: Nature, 356, 37-41 (1992). 